\title{
Study on the Indicators of Taiwanese Tour Guides' Service Quality
}

\author{
Jennifer Chen-Hua Min, Shu-Hua Chen, Mou-Yi Kao, Fu-Nan Hsu
}

Department of International Business, Ming Chuan University

Email: jennifer_min.tw@yahoo.com.tw, shchen@mail.mcu.edu.tw,mykao@mail.mcu.edu.tw,n32330015@yahoo.com.tw

Received June, 2013

\begin{abstract}
The tourism industry is considered a typical service industry, one in which the involvement of service components is relatively high. Serving and satisfying customers are fundamental goals of the tourism business, with service providers being part of the product itself. Given tour guides' roles as intermediaries between tourists and an unfamiliar environment, special attention should be paid to the service quality of tour guides, as it has the potential to increase tourist satisfaction and produce measurable benefits in profits and market share. The aim of the study is therefore to build the quality indicators of tour guide services. In terms of research methodologies, a combination of qualitative and quantitative approaches was conducted to reach the objectives. After both in-depth interviews and two rounds of focus group sessions, six dimensions (reliability, response, physical environment, guarantee, care and culture) and 30 indicators were obtained. Analytic Hierarchy Process (AHP) was applied to determine the weighting of various evaluation criteria on the indicators of Taiwanese tour guides' service quality. Among the criteria, "The tour guides have a precise service attitude and execution ability." was found to be the most important, while "During the trip, the tour guides dress properly." ranked as the least important.
\end{abstract}

Keywords: Tour Guides; PZB Model; Focus Group; AHP

\section{Introduction}

The significant role of service quality for business success has been well acknowledged. Delivery of high service quality can help organizations gain a competitive advantage and differentiate themselves more effectively in the marketplace [1]. However, service quality is rather difficult to measure because of the complex nature of services and unique qualities of a service transaction (i.e. heterogeneity, intangibility, and inseparability of production and consumption), while the quality of a physical good is relatively straightforward [2].

As an industry, the tourism industry is considered a typical service industry, one in which the involvement of service components is relatively high. Serving and satisfying customers are fundamental goals of the tourism business [3], with service providers being part of the product itself [4].

However, although extensive literature has been devoted to the service quality in the tourism field, there has been relatively little discussion of the service quality of the tour guide population, nor has there been any assessment of their quality of performance. The reason for this gap may be the lack of appropriate service quality indicators for tour guides. This is a worthwhile area of research, as tour guides play an important role in the success or failure of a tour experience and often crucially influence tourists' perceptions of the host destination [5]. Given tour guides' roles as intermediaries between tourists and an unfamiliar environment, special attention should be paid to the service quality of tour guides, which has the potential to increase tourist satisfaction and produce measurable benefits in profits and market share [6]. A combination of qualitative and quantitative approaches was conducted to reach the objectives. Since the Analytic Hierarchy Process (AHP) method systematizes complicated problems, is easy to operate, and integrates most of the experts' and evaluators' opinions, AHP method was therefore applied to determine the weighting of various evaluation criteria [7].

Hence, the purpose of this study is threefold:

1) Build the dimensions on the quality indicators of tour guide services

2) Construct related evaluation indicators on tour guide service quality

3) Evaluate the applicability of the quality indicators of tour guide services in the tourism industry.

\section{Methodology}

In terms of research methodologies of the current study, 
a combination of qualitative and quantitative approaches was conducted to reach the objectives. In terms of quailtative method, both in-depth interviews and two rounds of focus group sessions were carried out to ensure the inclusion of an adequate and representative set of indicators. Besides reviewing, comparing and contrasting relevant research literature, one-on-one interviews of tour guides were conducted to obtain information from their different points of view using open-ended questions. Both the interviews and focus group sessions were audio tape-recorded, and a content analytic approach was employed which provides the researchers with the opportunity to double check the answers and avoid missing any important information [8]. Then, a panel of experts including tour guides, practitioners, travel agents, and government officials in charge of tourism affairs were examine the generated list of service quality dimensions and criteria of tour guides to ensure that they adequately cover the most important aspects.

In the current study, indicators of tour guides' service quality involve many complex aspects and could be viewed as a multi-criteria decision-making problem. Therefore a systematic measurement was adapted to simplify the complexity and incorporate correlative criteria for analysis of issues. Since AHP method has the characteristics that is systematizes complicated problems, is relatively easy to operate, and integrates most of the experts' and evaluators' opinions, this study therefore adopted AHP for the contrivance of weights. For the quantitative method of the study, AHP was therefore applied to determine the weighting of various evaluation criteria on the indicators of Taiwanese tour guides' service quality.

AHP was first developed by Thomas L. Saaty in 1980 [7], and now has been applied in many diverse areas of social management sciences. In the 1990's, the tourism scholars also applied in tourism planning, evaluation, and decision making [9]. The method decomposes complicated problems from higher hierarchies to lower ones. Furthermore, it also systematizes the problem by utilizeing the subsystem perspective endowed in the system that can be easily comprehended and evaluated. Finally, it determines the priorities of the elements at each level of the decision hierarchy and synthesizes the priorities to determine the overall priorities of the decision alternatives. To apply AHP in prioritizing indicators of tour guides' service quality in this study, all indicators have to be structured into different hierarchical levels. This study shows the three-level hierarchy for indicators based on the hierarchical structures of AHP.

\section{Basic Concept of AHP}

\subsection{Hierarchical Structures}

Suppose there is a hierarchical structure showed in Fig- ure 1. Nodes in the hierarchy represent criteria, sub-criteria, or alternatives to be prioritized, and arcs reflect relationships between the nodes in different levels. Each relationship (arc) represents a relative weight or importance of a node at Level L relating to a node at Level L-1, where $\mathrm{L}=2,3, \ldots, \mathrm{N}-1, \mathrm{~N}$. The nodes at Level $\mathrm{L}$ do not necessarily connect to all the nodes at Level L-1, where $\mathrm{L}=2,3, \ldots, \mathrm{N}-1, \mathrm{~N}$.

The computation of weights is performed in the following way. Suppose there is a set of $n$ criteria $C=\left\{c_{L, 1}, c_{L, 2}, \cdots, c_{L, n}\right\}$ located at a hierarchical Level $\mathrm{L}$. Assuming that all the criteria at Level $\mathrm{L}$ are comparable with each other, $n(n-1) / 2$ paired comparisons of the $n$ criteria at Level $\mathrm{L}$ are performed. For each pair of comparisons, a decision maker (individual or group) uses the nine-point scale to reflect the degree of preference. The final AHP result is an assignment of weights to the criteria or alternatives at the lowest Level $\mathrm{N}$.

For the research, the word "criteria" may represent any one of three conceptual levels: identified usability dimensions, sub-dimensions, and individual questionnaire items. For example, in the lowest level (Level N), criteria can represent the set of individual questionnaire items, and criteria can represent the set of sub-dimensions in the Level $\mathrm{N}-1$. The top level node represents construct of overall usability which should ultimately be measured

\subsection{Pairwise Comparison}

In terms of the scales for quantifying pairwise comparesons, several approaches are available; although Saaty's [10] linear scale was the first proposed and has been used pervasively. Based on the fact that most humans cannot simultaneously compare more than seven objects (plus or minus two), Saaty [10] established 9 as the upper limit of the scale and 1 as the lower limit.

\subsection{AHP Data Analysis Procedure}

Using any of the scales the preference or dominance measures of paired comparisons are placed in a matrix form in the following manner:

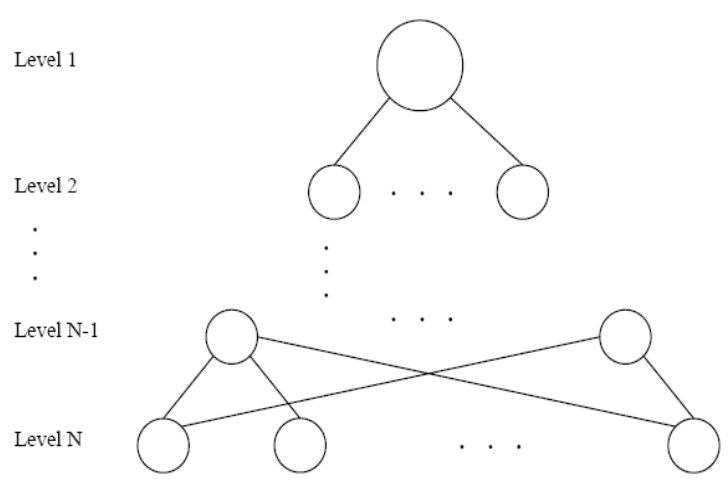

Figure 1. AHP structure. 


$$
M=\left[\begin{array}{cccc}
1 & m_{12} & \ldots & m_{1 n} \\
\frac{1}{m_{12}} & 1 & \ldots & m_{2 n} \\
\vdots & \vdots & \ldots & \ldots \\
\vdots & \vdots & \ldots & \ldots \\
\frac{1}{m_{1 n}} & \frac{1}{m_{2 n}} & \ldots & 1
\end{array}\right]
$$

Each $m_{i j}$ of the matrix represents the ratio by which criteria $i$ dominates criteria $j$. As mentioned in the previous section, criteria can be usability dimensions, subdimensions, or questionnaire items. Since $\mathrm{M}$ is a reciprocal matrix, in which each element of the lower-left diagonal part is the inverse of each element of upperright diagonal part, each $m_{i j}$ follows the specifications such as

$$
\begin{gathered}
m_{j i}=\frac{1}{m_{i j}}, m_{i j} \neq 0 \\
m_{i j}=1, \text { for } i=j \text { and } i, j=1,2, \cdots, n .
\end{gathered}
$$

To calculate weights based on the pairwise judgments, it is assumed that exact measurement was made so that each element can be decomposed into a ratio of weights as follows:

$$
m_{i j}=\frac{w_{i}}{w_{j}}
$$

Then the matrix $\mathrm{M}$ is expressed as

$$
M=\left[\begin{array}{cccc}
\frac{w_{1}}{w_{1}} & \frac{w_{1}}{w_{2}} & \cdots & \frac{w_{1}}{w_{n}} \\
\frac{w_{2}}{w_{1}} & \frac{w_{2}}{w_{2}} & \cdots & \frac{w_{2}}{w_{n}} \\
\vdots & \vdots & \cdots & \vdots \\
\frac{w_{n}}{w_{1}} & \frac{w_{n}}{w_{2}} & \cdots & \frac{w_{n}}{w_{n}}
\end{array}\right]
$$

Since $m_{i j}=\frac{w_{i}}{w_{j}}$ as defined above, $w_{i}=m_{i j}$ For all $j$, $w_{i}$ can take the general form of $w_{i}=\frac{1}{n} \sum_{j=1}^{n} m_{i j} w_{j}$, which leads to $m w_{i}=\sum_{j=1}^{n} m_{i j} w_{j}$. This expression can be denoted in matrix form as

$$
n w_{i}=A w_{j}(\text { or } A x=\lambda x)
$$

where $n$ (or $\lambda$ ) is the eigenvalue and $w$ is the eigenvectors. However, this is not solvable since there exist multiple eigenvalues and eigenvectors. Since $m_{i j}=1$ for all $i$, then $\sum_{i=1}^{n} \lambda_{i}=n$. If $\mathrm{M}$ is a consistent matrix (see the next paragraph), small variations of $m_{i j}$ keep the largest eigenvalue close to $n$, and the remaining eigenvalues close to zero. Therefore, the priority vector can be obtained from a vector $w$ that satisfies

$$
A w=\lambda_{\text {max }} w
$$

The vector $w$ is the eigenvector corresponding to the maximum eigenvalue. Where Saaty's (1995) provided 4 types of methods to solve eigenvalue

$$
w_{i}=\frac{1}{n} \sum_{j=1}^{n} \frac{a_{i j}}{\sum_{i=1}^{n} a_{i j}} \quad i, j=1,2, \cdots, n
$$

$$
w_{i}=\frac{\sum_{j=1}^{n} a_{i j}}{\sum_{i=1}^{n} \sum_{j=1}^{n} a_{i j}} \quad i, j=1,2, \cdots, n
$$

$$
w_{i}=\frac{\left(\prod_{j=1}^{n} a_{i j}\right)^{\frac{1}{n}}}{\sum_{i=1}^{n}\left(\prod_{j=1}^{n} a_{i j}\right)^{\frac{1}{n}}} \quad i, j=1,2, \cdots, n
$$

$$
w_{i}=\frac{\left(\frac{1}{\sum_{i=1}^{n} a_{i j}}\right)}{\sum_{j=1}^{n}\left(\frac{1}{\sum_{i=1}^{n} a_{i j}}\right)} \quad i, j=1,2, \cdots, n
$$

To obtain relative weights, the sum of which is equal to one, the eigenvector should be normalized in the following manner:

$$
w^{\prime}=\left[\frac{1}{\sum_{i=1}^{n} w_{i}}\right] w
$$

\section{Results and Conclusions}

After interviewing and two round focus groups, six dimensions (reliability, response, physical environment, guarantee, care and culture) and 30 indicators were obtained. A panel of 25 experts including tour guides, prac- 
titioners, and tourism professors were collected. The results from the questionnaires were calculated in sequence to derive the weightings and then the various weight alternatives were evaluated. The outcomes were then prioritized by weight, and the expert opinions were combined to form the tour guides' service quality. Each weighting mode derived from this research was consistent as all CI's (Consistency Indicators) and CR's (Consistency Rates) were below 0.1. Among the six dimensions, reliability (28.2\%) is the most important evaluation framework, and the culture (7.5\%) is the least important. Among the criteria, "The tour guides have a precise service attitude and execution ability." was found to be the most important, while "During the trip, the tour guides dress properly.” ranked as the least important.

The present study contributes to the field of knowledge about the importance of service quality in the tourism industry, specifically in regards to tour guides. It is helpful if future work can build upon this study's framework and develop scales of service quality of tour guides from different tourists' perspectives. The results of the current study also can help travel practitioners and tour guides properly allocate resources, improving and tailor-making services to meet the expectations of Taiwan inbound tourists.

\section{Acknowledgements}

This research was funded by a research grant from the National Science Council in Taiwan (NSC 101-2410H-130-061-).

\section{REFERENCES}

[1] O. M. Karatepe, U. Yavas and E. Babakus, "Measuring Service Quality of Banks: Scale Development and Vali- dation,” Journal of Retailing and Consumer Services, Vol. 12, pp. 373-383, 2005.

doi:10.1016/j.jretconser.2005.01.001

[2] A. Parasuraman, V. A. Zeithaml and L. L. Berry, “A Conceptual Model of Service Quality and Its Implications for Future Research,” Jounal of Marketing, Vol. 49, 1985, pp. 41-50. doi:10.2307/1251430

[3] J. Min, V. Tang and M. S. Yin, "Prioritising the Emotional Intelligence (EI) Needs of Tourism Undergraduates in Taiwan,” Journal of Hospitality Leisure Sport Tourism Education, Vol. 10, 2011, pp. 14-29. doi:10.3794/johlste.102.351

[4] S. Langhorn, "How Emotional Intelligence can Improve Management Performance," International Journal of Contemporayy Hospitality Management, Vol. 16, 2004, pp. 220-230. doi:10.1108/09596110410537379

[5] J. Min, “A Short-form Measure for Assessment of Emotional Intelligence for Tour Guides: Development and Evaluation,” Tourism Management, Vol. 33, 2012, pp. 155-167. doi:10.1016/j.tourman.2011.02.014

[6] E. W. Anderson, C. Fornell and D. R. Lehmann, "Customer Satisfaction, Market Share, and Profitability: Findings from Sweden,” Journal of Marketing, Vol. 58, 1994, pp. 53-66.doi: $10.2307 / 1252310$

[7] T. L. Saaty, "The Analytic Hierarchy Process: Planning, Priority Setting,” New York: McGraw Hill International Book Co., 1980.

[8] M. K. Brady and J. J. Cronin, "Some New Thoughts on Conceptualizing Perceived Service Quality: A Hierarchical Approach,” Journal of Marketing, Vol. 65, 2001, pp. 34-49. doi:10.1509/jmkg.65.3.34.18334

[9] C. Ryan, "Recreational Tourism- A Social Science Perspective,” London: Routledge, 1991.

[10] T. L. Saaty, "Decision Making for Leaders: The Analytic Hierarchy Process in a Complex World,” RWS Publications, 1995. 